Mots. Les langages du politique

\title{
Discours médiatiques post-attentats : une perspective historique (1995-2016)
}

Media narratives after terrorist attacks: a historical perspective (1995-2016) Discursos mediáticos post-atentados: una perspectiva (1995-2016) histórica

Isabelle Garcin-Marrou et Isabelle Hare

\section{OpenEdition}

Journals

Édition électronique

URL : https://journals.openedition.org/mots/23726

DOI : $10.4000 /$ mots. 23726

ISSN : 1960-6001

Éditeur

ENS Éditions

Édition imprimée

Date de publication : 5 novembre 2018

Pagination : 19-35

ISBN : 979-10-362-0082-3

ISSN : 0243-6450

\section{Référence électronique}

Isabelle Garcin-Marrou et Isabelle Hare, « Discours médiatiques post-attentats : une perspective historique (1995-2016)», Mots. Les langages du politique [En ligne], 118| 2018, mis en ligne le 05 novembre 2020, consulté le 23 avril 2022. URL : http://journals.openedition.org/mots/23726 ; DOI : https://doi.org/10.4000/mots. 23726 


\section{Discours médiatiques post-attentats : une perspective historique (1995-2016)}

Les événements terroristes survenus en France et en Europe depuis 2015 ont relancé la réflexion sur les réactions des sociétés touchées - révolte, recueillement, rassemblement - comme sur les discours post-attentats, émanant des médias, du gouvernement ou de la classe politique. La question des relations entre médias et terrorisme a déjà donné lieu à de nombreuses contributions (Wieviorka, Wolton, 1987; Schlesinger, 1991; Garcin-Marrou, 2001 ; Hare, 2007), pour n'en citer que quelques-unes. Des travaux se sont penchés sur les conséquences géopolitiques et médiatiques du 11 septembre 2001 en matière de terrorisme (Lits éd., 2004 ; Derrida, Habermas, 2004 ; Dayan éd., 2006 ; Niemeyer, 2011); d'autres ont contribué à la compréhension des représentations médiatiques des attentats survenus en France dans cette dernière décennie (Lefébure, Sécail éd., 2016 ; Truc, 2016).

La réitération des actes terroristes sur le sol européen a conduit à interroger la permanence des relations entre État et médias (Hare, 2016), à questionner les enjeux sociopolitiques des discours des médias (Garcin-Marrou, 2018), réactivant parfois l'idée d'un cadrage discursif dominant, celui d'un «11 septembre européen » (Truc, 2016). Ce sont précisément les discours médiatiques de consensus, relatifs à l'illégitimité de la violence terroriste et à la légitimité des actions de réparation - celles qui émanent de l'exécutif producteur de symboles visant à restaurer l'ordre émotionnel, social et international (Boussaguet, Faucher, 2016), que la contribution entend étudier en repartant de travaux consacrés aux médias et au terrorisme depuis 20 ans.

Le regard proposé est comparatif et prend pour point de départ l'attentat du 25 juillet 1995 à Paris, à la station Saint-Michel du RER B (7 morts et 84 blessés), et de l'attentat du 3 décembre 1996, à la station Port-Royal du RER C (4 morts et 91 blessés), pour envisager ensuite les attentats de Charlie Hebdo le 7 janvier 2015 (17 morts et 11 blessés), ceux du Stade de France, des terrasses parisiennes et du Bataclan le 13 novembre 2015 (137 morts et 350 blessés) et celui

Univ Lyon, Sciences Po Lyon, ELICO

isabelle.garcin-marrou@sciencespo-lyon.fr

Univ Lyon, université Lumière Lyon 2, ELICO

i.hare@univ-lyon2.fr

Mots. Les langages du politique $\mathrm{n}^{\circ} 118$ novembre $2018 \bullet 19$ 
de la promenade des Anglais à Nice le 14 juillet 2016 (86 morts et 450 blessés). Quand l'ombre de la barbarie plane sur la société, quelles sont les répercussions symboliques, sociales et politiques du terrorisme, et comment les médias organisent-ils la «réponse» aux attentats? Comment les récits médiatiques articulent-ils les figures de l’État, de la société civile et, enfin, des auteurs des violences, assimilés, de façon contemporaine, à un «ennemi intérieur»? Depuis les attentats de 1995 et 1996 à Paris jusqu'en 2001 (New York), 2004 (Madrid) et 2005 (Londres), les auteurs d'actes terroristes ont en effet été identifiés comme des "étrangers» (à la société, à l'espace public et à la démocratie). Or les événements de 2015 et 2016 (Paris, Nice) impliquent des jeunes qui ont grandi, pour certains, dans des écoles de la République française. Ces questions constituent les fils directeurs de la comparaison, qui met en regard des événements d'ampleurs très différentes tout en s'efforçant de comprendre, au-delà de ces spécificités, les récits médiatiques et leurs mises en scène des figures étatiques, sociales, civiles.

Le corpus constitué est composé des titres suivants pris dans leurs trois éditions parues après chaque attentat : pour 1995 et 1996, Le Monde (LMon), Libération (Lib), Le Figaro (LFig), France-Soir (FSoir), le quotidien régional Sud Ouest (SO) et l'hebdomadaire Le Point (LPo) ; pour 2015 et 2016, les mêmes journaux, auxquels nous ajoutons Aujourd'hui en France (AeF) et L'Express (LExp), puis les quotidiens belge Le Soir (LSoir) et américain The New York Times (NYT). Ces deux titres participent à la mise en sens des violences, du fait de la nationalité belge de plusieurs auteurs des attentats de novembre 2015 et du renvoi à la symbolique du 11 septembre 2001.

Nous avons choisi de travailler à partir des versions papier de ces titres, afin de pouvoir appréhender le dispositif médiatique dans son intégralité et de façon égale, puisqu'il n'existait pas de version en ligne de ces médias pour 1995 et 1996. L'analyse proposée vise à identifier les grandes tendances discursives à l'œuvre dans le temps chaud "post-attentats "1. Nous repérons les lexiques employés dans les différents titres; cette analyse de contenu se double d'une étude de l'énonciation qui permet de voir comment se dessine la frontière entre le «nous», le «vous» et le «eux» dans ces moments de crise. Les différentes analyses appréhendent donc chaque événement sous l'angle de leur traitement médiatique. Indiquons enfin que les analyses (lexicales, énonciatives, narratives) ont été menées de façon systématique sur l'ensemble du corpus, mais que seuls les éléments saillants sont cités dans cet article².

1. Le corpus inclut les éditions des quotidiens parues du lendemain au troisième jour suivant chaque attentat et l'édition immédiatement suivante pour les hebdomadaires.

2. Précisons que tous les extraits cités dans cet article sont issus des discours des journaux analysés - lesquels reprennent parfois les discours des responsables politiques. 


\section{L’État face au terrorisme : l'incarnation de la sécurité}

Sans revenir sur les nombreuses définitions du terrorisme, il est une caractéristique commune aux actes répertoriés comme relevant du «terrorisme»: ils visent à semer la terreur et à marquer les opinions publiques tout en établissant comme destinataires finaux de leurs actes les États (les instances et les pouvoirs politiques) et les sociétés (les citoyens). Le terrorisme cherche à déstabiliser les structures étatiques, sociales et le public, mais porte atteinte principalement, du fait d'une asymétrie actancielle majeure, à celles et ceux qui composent l'ensemble sociopolitique : les membres de l'espace public touché par la violence. La visée explicite du terrorisme touche à la légitimité des pouvoirs, notamment à celle liée à la garantie de la sécurité des citoyens - c'est là tout le sens que Thomas Hobbes donne à l'existence même du Souverain (Hobbes, 1971 [1651]). L'usage du monopole de la violence légitime, pour rétablir l'ordre et la sécurité, est également questionné. Face à cette mise en cause principielle de l'État démocratique, dont la marge de manœuvre est encadrée par le droit, l'un des éléments importants que l'on observe dans les discours médiatiques tient en cette mise en mots et en sens de la rupture sécuritaire provoquée par l'acte terroriste et de la réaffirmation de l'existence et de l'action de l'État.

\section{Saint-Michel et Port-Royal : légitimité, unité politique et émotion maîtrisée}

En 1995 et 1996, les articles sur les attentats entrent dans le chemin de fer usuel de la plupart des journaux - sauf pour France-Soir, qui propose des dossiers fournis - et ne provoquent pas de rupture dans le continuum éditorial usuel des médias 3 . La réaction des pouvoirs d’État constitue un élément central du récit médiatique post-attentats et répond à la demande sécuritaire des citoyens et de la société. Lors de l'attentat de Saint-Michel, Le Monde convoque en une «le gouvernement», tandis que Libération décrit la visite du ministre des Transports, Bernard Pons, à Saint-Michel, et relaie en titre le message du ministère de l'Intérieur lançant un "appel à la vigilance», mais demandant de ne pas «céder à la psychose». Le Figaro décrit la gestion de crise mise en place par le président de la République (Jacques Chirac) et le ministre de l'Intérieur (JeanLouis Debré). Le quotidien, proche de la majorité de gouvernement, propose

3. L'Express ne propose pas d'article sur l'attentat dans son édition du 27 juillet 1995 . Les collections d'août 1995 et décembre 1996 étant indisponibles à la BnF, nous n'avons pas pu vérifier si l'hebdomadaire avait produit du contenu sur ces attentats. L'absence d'article dans l'édition parue deux jours après l'attentat de Saint-Michel s'explique sans doute par le fait qu'elle a été imprimée avant le drame. 
par ailleurs de très nombreuses photographies des responsables politiques au visage - fermé - de circonstance. Le Point mentionne le déplacement immédiat sur le lieu de l'attentat de J. Chirac, affichant « une mine sombre», et des ministres de l'Intérieur, de la Justice et des Transports. En 1996, Libération propose une photographie du Premier ministre et du ministre de l'Intérieur, du préfet et du maire de Paris sur les lieux de l'attentat; le surtitre indique que «le plan Vigipirate a été rétabli ». Tous les journaux rappellent donc que, face à la violence, le pouvoir est présent, agit et n'est pas seulement une affaire de mots. Face au trouble majeur que constitue la violence terroriste, les médias réaffirment donc l'existence du politique en mettant en discours le pouvoir incarné, au sens hobbesien du terme, dont l'impératif premier est de garantir la sécurité des membres du corps politique (Garcin-Marrou, 2001; Guigo, 2016).

Dans les discours cités par les médias, les champs lexicaux sont convergents; les pouvoirs publics réaffirment l'autorité de l'État et sa capacité à agir : les verbes déclaratifs sont nombreux - "déclarer», "affirmer», "dire» - et ces verbes s'appliquent aux mesures immédiates et visibles de la lutte contre les «terroristes» (immédiatement qualifiés comme tels par les responsables politiques et par les médias); la «détermination» dans la «lutte par tous les moyens contre le terrorisme» est martelée dans les discours ; l'État s'affirme comme l'instance susceptible de repousser la «barbarie », la «lâcheté » ou les «assassins aveugles». Le "plan Vigipirate», créé en 1995, est mentionné de façon très fréquente comme instrument concret de présence de l'État, par les moyens policiers et militaires déployés. Le premier élément saillant des discours de 1995 et 1996 tient donc en la réaffirmation de l'autorité du pouvoir face au terrorisme.

Les discours décrivent - construisent - par ailleurs une relative unanimité partisane. Sous un gouvernement de droite (celui d'Alain Juppé), les déclarations relatives à l'identification et au rejet de l'ennemi extérieur convergent. En 1995, Le Monde et Le Figaro proposent chacun un encadré («Les réactions » et "Le monde politique horrifié ») qui rassemble les propos du Premier ministre, du premier secrétaire du Parti socialiste (Henri Emmanuelli), du secrétaire national du Parti communiste français (Robert Hue), des écologistes, du cardinal Lustiger, des syndicats des transports parisiens. Aucune dissonance interprétative dans les propos cités, de la droite à la gauche communiste, tous rappellent la «lâcheté » de l'acte, la «nécessaire fermeté de l’État » et - surtout - le fait que «la communauté nationale doit se montrer unie et solidaire». Seul le Front national est cité comme marquant sa différence; le parti pointe ainsi «la dégradation des conditions de sécurité ». À cette exception notable près, l'ensemble des responsables politiques se retrouve dans ce que le premier secrétaire du Parti socialiste énonce, c'est-à-dire une unité politique attendue face à la violence qui déstabilise la société. En 1995, il faut noter la mention médiatique de l'appel lancé à la société civile par le ministre de l'Intérieur, 
J.-L. Debré, qui déclare souhaiter « un rassemblement de tous les citoyens pour lutter contre le terrorisme ». En 1996, l'État et le gouvernement Juppé ont appris de 1995, les logiques de gestion de la crise sont rodées, et ce sont la fermeté et la légitimité de la lutte contre le terrorisme - «par tous les moyens» selon le président J. Chirac - qui s'imposent comme axe discursif politique et médiatique majeur. Face à cette unanimité, Libération pose la question suivante : "le consensus politique autour de l'attentat va-t-il durer? » Si aucune déclaration contradictoire ne ressort de son discours, le quotidien pointe un «consensus presque sans fausses notes » et " une unité nationale presque retrouvée». France-Soir salue le «signe réconfortant» de la "solidarité face aux tueurs» des «politiques [qui] toutes tendances confondues - à l'exception du Front national - ont assuré le gouvernement de leur soutien », tandis que Libération revient sur les accommodements des pouvoirs d'État avec le terrorisme (corse). L'attentat de 1996 ne suspend donc pas les analyses politiques et critiques, et si la figure narrative des gouvernants est sanctionnée positivement dans sa gestion immédiate de l'attentat, les oppositions partisanes ne disparaissent pas complètement de la narration médiatique.

Il faut remarquer, enfin, les émotions manifestées par les membres de l'exécutif. Elles sont notamment décrites par Libération («Bernard Pons accueille Alain Juppé. [Ils] sont bientôt rejoints par le président de la République. Peu de déclaration. L'émotion, le silence») ou par Le Figaro ("L'émotion de JeanLouis Debré »). En 1996, même retenue. Les émotions sont montrées dans les médias, par les images des «mines sombres» des responsables politiques, et exprimées par des formules de compassion envers les victimes, mais ces émotions passent surtout par la dénonciation de la «barbarie ». L'émotion de l'État est donc très maîtrisée et les responsables restent dans le registre de l'autorité, à tel point que l'éditorial de France-Soir se clôt, en décembre 1996, par un reproche adressé à l'exécutif : « manque juste ici un sentiment très partagé cette nuit : la colère ». Face au terrorisme, les médias proposent donc, de façon dominante, le récit de responsables politiques maîtrisant leurs émotions et mettant en œuvre, avant tout, la prise en charge des victimes et la lutte contre les auteurs de la violence.

\section{Charlie Hebdo, 13 novembre et Nice : de l'unité politique à la désunion électoraliste}

Les attentats survenus en 2015 produisent une forme de consensus politique - au moins dans les premiers jours - quant à la réaction de l'exécutif. Néanmoins, au fil des actes, ce consensus apparaît de plus en plus fragile et de courte durée. Dans les discours médiatiques, la légitimité de l'action gouvernementale face au terrorisme bascule progressivement vers une relative illégitimité. 
Lors des deux vagues d'attentats de 2015, la violence terroriste est qualifiée successivement d'atteinte à la liberté, comme l'indiquent en une Le Figaro ("La liberté assassinée») et Aujourd'hui en France ("Ils ne tueront pas la liberté »), puis d'acte guerrier en novembre. Au lendemain du 13 novembre 2015, comme l'a montré Marie Veniard dans son travail portant sur l'usage du terme guerre dans Le Monde et Le Figaro (Veniard, 2018), le champ lexical guerrier développé par F. Hollande4 ("Ce qui s'est produit [...], c'est un acte de guerre») pose un cadre repris par la plupart des médias : «La guerre en plein Paris» (LFig), «Comment gagner la guerre» (LExp), «Notre guerre» (LPo).

L'attaque terroriste implique une réponse de l'État à la hauteur de l'atteinte (Wieviorka, Wolton, 1987), comme le déclare la une du Figaro au lendemain du 13 novembre: «Hollande face au défi de la riposte». Quelle que soit la posture choisie par l'État, les médias construisent la représentation de l'action gouvernementale, en exprimant solidarité ou désolidarisation vis-à-vis de celleci, directement ou par l'intermédiaire de propos politiques rapportés. L'injonction médiatique est forte, comme l'atteste le titre «François Hollande face à son 11 septembre» $(A e F)$ : qualifier ainsi l'attaque survenue à Charlie Hebdo suppose une réponse de l'État à la hauteur de celle des États-Unis en 2001. Mais la question de l'unité nationale et politique derrière F. Hollande préoccupe davantage les médias une fois les terroristes mis « hors d'état de nuire» (LFig). En effet, rapidement quotidiens et magazines francophones interrogent tous, à des degrés divers, "Le casse-tête de l'union sacrée» (AeF) et évoquent «Une union nationale à géométrie variable » $(L i b)$, «à marche forcée » $(L i b)$, se proposant d'analyser ce qui se passe «derrière l'unité nationale» (LExp). En dépit du succès populaire de la marche républicaine du 11 janvier, la légitimité de l'action de l'État est donc rapidement interrogée par la presse.

Les attentats du 13 novembre renforcent ce phénomène et, passées les premières heures des attaques, la question de l'unité nationale ressurgit, produisant d'abord ce que Libération qualifie d' "Union malgré les tentatives de récupération », Le Monde soulignant, lui, «des voix dissonantes chez les politiques». Par ailleurs, "Les Républicains réclament des comptes» $(A e F)$ et Le Point observe que «F. Hollande peut retrouver une légitimité». Dans ces moments de tensions et d'émotions, des joutes verbales entre leaders politiques apparaissent, destinées à imposer leur vision de ce que doit être cette " union de circonstance» (LFig), et mettent ainsi en avant les dissensions lexicales internes chez Les Républicains, Nicolas Sarkozy préférant parler de « solidarité nationale » et François Fillon «d'union nationale ».

Après l'attentat de Nice, les médias s'emploient donc à comprendre comment une telle tragédie a pu survenir, en analysant le contexte de la tuerie, mais ils soulignent aussi la difficulté à cerner un ennemi venu désormais de l'inté-

4. Voir dans ce numéro l'article de Laurie Boussaguet et Florence Faucher (note des coordinateurs). 
rieur - isolé et visant des «soft targets». D’où la nécessité, énoncée par les journaux, de repenser ce qui est devenu avant tout un problème social et politique interne aux pays touchés. Comme le souligne The New York Times : «Ghettoization, polarization, and alienation in a country like France are profound. »

Par ailleurs, certains journaux évoquent le climat politique délétère qui règne en France : « déséquilibre de la paix civile », «illégitimité de l'action gouvernementale dénoncée par la droite» (LSoir). Ils notent la rapide transition vers les dissensions et les reproches politiques : «l'union sacrée qui avait prévalu dans les jours suivant les attentats de Charlie Hebdo et de l'Hyper Cacher n'est plus quant à elle qu'un lointain souvenir » (LMon). Le Figaro, très ferme à l'égard de l'exécutif, enjoint l'État, en une, à «Gagner la guerre », développant dès son éditorial le champ sémantique du combat, à la fois par les symboles convoqués ("la Marseillaise», «l'hydre islamique») et le vocabulaire mobilisé (« riposte », « réarmement législatif », « cinquième colonne »). Le Soir, enfin, évoque l'illégitimité d'une réaction militaire par un rappel de l'histoire française, «France, la guerrière devenue cœur de cible», renvoyant le pays à son passé belliqueux et colonialiste, et explique ainsi l'origine de ses maux et la présence d'un «ennemi intérieur» sur son sol.

L'émotion puis l'autorité, attendues par l'opinion publique et la classe politique, participent bien d'une forme de catharsis dans l'imaginaire collectif (Girard, 1994), la faute ne pouvant être expiée et l'émotion apaisée, après un attentat, que par la traque et la punition des coupables. Mais l'autorité de l'État - saluée à la suite des attentats de Charlie Hebdo et du 13 novembre est décrite dès le lendemain de la tuerie de Nice comme une forme d'autoritarisme de l'exécutif (ancré dans le triptyque "état d'urgence», «renseignement», « intervention militaire française à l'extérieur»).

La médiatisation de l'action de l'État au lendemain des attentats de 2015 constitue une injonction forte au politique, la population attendant une réaction du gouvernement face à «l'attaque». Pierre-Emmanuel Guigo (2016) analyse la communication de F. Hollande en janvier - proche de celle des attentats de novembre - et observe une attitude oscillant entre la figure de Père de la Nation - garant de la sécurité des Français - et celle d'omniprésident (au regard du nombre très important de ses interventions télévisées dans les semaines suivant les attentats), mais aussi celle de président silencieux (lors des marches républicaines du 11 janvier et de l'hommage auxvictimes aux Invalides le 27 novembre). Ces deux hommages relèvent de manifestations post-attentats « convoquées à un jour et à une heure précise (souvent par le gouvernement) » dont l'une des finalités serait « d'adresser un message aux terroristes et au reste du monde » (Truc, 2016). L'émotion du chef de l'État peut être ici considérée comme une émotion rationalisée et politisée. La proclamation du deuil national (un jour pour Charlie Hebdo et trois jours pour les attentats du 13 novembre et de Nice) détermine la gravité des événements et leur caractère exceptionnel. 
Depuis 2015, F. Hollande s'affirme comme un chef de guerre, mais cet autoritarisme est-il le plus à même de résoudre un problème de société? C'est ainsi que les journaux interrogent, au lendemain de l'attentat de Nice, la posture politique du président de la République et la pertinence des actions de l'État, «bravaches» et «inutiles» (LSoir). Le Point observe par ailleurs que le propos présidentiel s’inspire très fortement de la rhétorique militaire américaine, produisant un «discours de la vengeance ». L'inadéquation de la réponse politique à la menace terroriste apparaît ainsi comme un discours assez largement partagé par les médias de notre corpus.

\section{La société civile : des morts individuelles à la tragédie collective}

Face à la blessure terroriste, comment les médias prennent-ils en charge la douleur, les émotions, la rupture d'un des éléments constitutifs du social - la garantie politique de la sécurité? Quels peuvent être les discours adressés à une société en peine, dont certains des membres ont été atteints? Quelles sont les figures de cette entité, la "société civile», si présente dans les discours contemporains ? Là encore, la diachronie permet de tracer quelques évolutions importantes en vingt ans de discours médiatiques.

\section{Saint-Michel et Port-Royal : émotions et distance}

Les journaux de 1995 et 1996 proposent tous un récit à hauteur humaine ; les témoignages sont assez présents et les journaux rendent compte des blessures physiques et des mutilations des survivants blessés ou simplement hébétés. Du Monde du 27 juillet 1995, qui consacre une moitié de page aux témoignages ( «ll y a eu une lueur orangée. Puis une forte détonation. Les vitres ont volé en éclats »), à France-Soir dont l'édition du 4 décembre 1996 est emplie de ces témoignages et photographies de victimes, les récits des attentats ne cèlent rien de la douleur. Les lexiques sont nourris, variant d'intensité selon les journaux. Le Monde n'utilise pas de terme choc en titre, mais décrit les «corps brûlés », les «membres arrachés», les «polycriblages » et les «blessures de guerre». Libération évoque, en 1995, «la mort aveugle» dans le métro, «l'odeur de poudre», les «amputations», les «corps disloqués» et titre, en 1996, «Retour de terreur», racontant ensuite les « corps sans jambes», les «mares de sang ». Le Figaro mentionne dans ses titres le «carnage » ou le «massacre des Innocents», puis décrit les «membres arrachés », les personnes «blastées». France-Soir utilise un rubriquage, apposé en biais, «Carnage dans le métro». Notons un éditorial paru en page «Radio-Télévision» du Monde, titré «Attentat» et qui revient sur la médiatisation télévisuelle des attentats : 
L'œil guettait le sang : il n'en vit pas. [...] Au cours de la vague d'attentats de 1995, le chef de l'État s'était plaint d'une "dérive médiatique sans précédent» qui avait conduit les chaînes de télévision à passer en boucle les images les plus sanglantes. Rien de tel, ici.

L'atteinte faite à la société est donc dans une certaine mesure tenue à distance dans les images, et les journaux usent d'ensembles lexicaux qui disent l'atteinte aux corps, mais mentionnent très peu les émotions. Celles-ci sont pourtant présentes, de façon plus marquée dans France-Soir ou dans Le Figaro, mais elles ne constituent pas l'essentiel des discours, centrés sur les interrogations relatives aux responsables et sur le travail d'enquête. Les récits mettent en avant une figure cruciale, celle du « secours » dû aux personnes par l'État; le «plan rouge » (pour les pompiers) et le «plan blanc» (pour le SAMU) sont ainsi décrits par l'ensemble des journaux. Mais les discours n'opèrent pas ce que Claire Sécail décrit comme constituant, en janvier 2015, une «mise en scène de la mobilisation et des émotions» (Lefébure, Sécail éd., 2016) par la manifestation et la coprésence dans l'espace public. Les journaux de 1995 et 1996 ne mettent pas en scène cet ensemble social uni face au terrorisme. Les actes de solidarité sont décrits mais apparaissent très circonscrits. Ils émanent des cafetiers voisins des stations de RER touchées, des pharmaciens du quartier ou, encore, des usagers qui sont venus en aide au plus près des explosions. À la différence des discours des années 2015 et 2016, la société n'est pas constituée comme une entité souffrant à l'unisson des victimes directes, et, par exemple, les lieux des attentats ne sont pas constitués en mémoriaux civils. Comme cela sera le cas en 2001 et lors des attentats suivants, les journaux donnent les noms des personnes décédées; France-Soir propose quelques portraits précisant la façon dont les victimes sont décédées. Mais l'émotion représentée n'est pas construite comme collective, y compris dans France-Soir qui raconte - et montre - des tragédies individuelles.

\section{Charlie Hebdo, 13 novembre et Nice : de l'émotion crue à la résilience}

G. Truc observe que la monstration des conséquences d'un attentat (morts et blessés, dégâts matériels, secours) varie considérablement selon l'origine géographique du quotidien et sa proximité avec le lieu de l'attaque terroriste. Ainsi, lors des attentats de Madrid (mars 2004), médias espagnols et américains mobilisent des cadres iconographiques différents. Le chercheur explique que le fait de ne pas montrer de cadavres participe d'une rhétorique quasi guerrière :

[...] la grande majorité des journaux américains se sont de nouveau conformés à la règle (déjà présente lors des attentats du 11 septembre, ndlr) qui veut que, en temps de guerre, l'on ne montre pas les morts de son camp, encore moins à visage découvert. (Truc, 2016, p. 102) 
En 2015 et 2016, deux grands dispositifs éditoriaux structurent la monstration de "l'horreur»: d'un côté, la vision crue des amoncellements de corps drapés, des corps en morceaux (un pied qui dépasse, de la matière sur la chaussée), des lieux post-attentats, c'est le choix des hebdomadaires, d'Aujourd'hui en France et du New York Times. De l'autre, la majorité des quotidiens raconte les conséquences de l'attentat à travers des symboles (des poussettes abandonnées, des rescapés sortant de l'hôpital, présence des secours et des forces de l'ordre, pare-brise du camion incriminé, des bouquets de fleurs, des bougies).

Les mots disent aussi l'inacceptable, allant de la «stupeur» à «l'horreur». Au lendemain du 7 janvier, quatre modalités de une permettent de construire l'événement : la mise en danger de la liberté d'expression et de la presse, «lls ne tueront pas la liberté» $(A e F)$, «La liberté assassinée» (LFig) ; la collectivisation de la souffrance par l'emploi d'un «nous» inclusif, "Nous sommes tous Charlie» (Lib), «Nous sommes la France» (LExp); la mention d'un «chrononyme» (Bacot, Douzou, Honoré, 2008), «Le 11 septembre français» (LMon), sa construction, «7 janvier 2015 » (LPO), et l'ironie, « Morts de rire» (LSoir). Les articles développent l'idée qu'à travers les attentats à Charlie Hebdo et à l'Hyper Cacher, ce sont des symboles qui ont été visés. L'ampleur et la nature de la réaction médiatique à la suite de l'attentat de Charlie Hebdo s'expliquent par le fait que des journalistes ont été touchés : «Que le point de départ en ait été une affaire de presse n’y est sans doute pas étranger» (Nora, 2015, p. 5).

$\mathrm{Au}$ lendemain du 13 novembre, la presse internationale et nationale est unanime dans la condamnation et dans l'émotion. Le Monde titre «La terreur à Paris », d'autres quotidiens évoquent sous la même forme nominalisée l'ampleur des attentats : le «carnage» (Lib) ou encore le «massacre» (LSoir). Les éditions d'Aujourd'hui en France et du Soir précisent le caractère parisien - et centralement politique - de l'attentat : «Massacre terroriste en plein Paris », «Massacre à Paris ». D’autres font de ces attentats une déclaration de guerre et de représailles : «La guerre en plein Paris » (LFig), "Comment gagner la guerre » (LExp). Seul le New York Times caractérise en une la gravité des attentats par le nombre de victimes, "Paris terrorist attacks kill over 100 » (Koren, 1996).

Deux registres discursifs référant à la société civile sont à noter : le premier renvoie au caractère inédit des attaques (modalité, gravité) par les expressions «carnage » ou « attentat sans précédent », " dans une autre dimension », « 3 heures de terreur pour un carnage»; par la qualification de l'ampleur de la tragédie, «une nuit d'horreur», «massacre coordonné», «barbarie terroriste », «l'enfer de Dante»; par la comparaison implicite avec les attentats de Charlie Hebdo, "une marche de plus dans l'horreur», "même si l'horreur est décuplée par rapport à janvier». Le deuxième registre est celui de l’hyperbole incluse dans la parole testimoniale : les blessés (Salomé 15 ans, un couple Benjamin et Célia, des voisins) font le récit cru de leur soirée, et racontent ce 
que les images ne peuvent ou ne doivent pas montrer : «le sang gicle partout autour d'eux», "des éclats de cervelle et de chair» (LFig). Un médecin affirme «n'avoir jamais vu ça» (Lib). Les propos rapportés accréditent la réalité des événements et signifient leur caractère tragique.

L'attentat de Nice génère le même type de qualification médiatique, qui poursuit la narrativisation de la tragédie. Les unes disent «L'horreur à Nice», «La terreur - Un 14 juillet » et l'incompréhension : «Pourquoi? ? (Lib), «C'étaient des enfants» $(A e F)$. D'autres situent l'attentat dans une continuité dramatique : "L'horreur à nouveau » et «Gagner la guerre » (LFig). Présents par le discours rapporté, les témoins et les victimes soutiennent l'expression du pathos. Les articles racontent des moments de tragédie : "scènes d'horreur », «il tenait son petit garçon de deux ans sans vie dans ses bras» (LFig), «des corps jonchent le sol [...] certains inertes, d'autres hagards... », "des morts tout le long de la route » (LMon), «des corps partout», «du sang " (LSoir), "soft targets », "simply a crowd celebrating in the street», "scenes of pandemonium » (NYT). Mais ce sont aussi des scènes d'apocalypse : «j'ai vu des corps voler comme des quilles de bowling sur son passage. Entendu des bruits, des hurlements que je n'oublierai jamais», " des corps tous les 5 mètres, sans vie, des membres [...] Des gémissements », " chaos absolu », " des scènes de carnages », " une scène de guerre » (LMon), " a vast tableau of carnage and panic» (NYT).

Enfin, il faut mentionner brièvement les portraits (Wrona, 2005). Après les attentats du 11 septembre 2001, le New York Times avait initié les portraits nécrologiques (portraits of grief), produisant ce que G. Truc nomme «la singularisation des victimes» (Truc, 2016, p. 260), nécessaire à la «compassion ». Les attentats de Charlie Hebdo déclenchent la litanie mortuaire dans les médias, qui s'ouvre par le portrait (verbal et visuel) de ces douze victimes, parmi lesquelles figurent certes des noms connus, mais aussi des anonymes - le garde du corps, le policier, etc. - que ces portraits désanonyment : "Ils s’appelaient Frédéric, Ahmed et Mustapha» $(\mathrm{AeF})$. Ce dispositif est reconduit après le 13 novembre dans tous les médias, à l'exception notable des hebdomadaires. Après l'attentat de Nice, les médias construisent des figures victimaires archétypales : une famille du Nord décimée (six victimes), un jeune garçon de quatre ans (photographié sur une plage), une femme marocaine de 60 ans (Fatima) - première victime du tueur - et un jeune homme qui s'est sacrifié pour sauver sa femme enceinte. La répétition crée alors un effet dramatique et accentue le caractère tragique de l'événement.

La succession d'attentats entre 2015 et 2016 génère ainsi une forme de ritualisation dans la gestion des émotions civiles post-attentats, reprise mais aussi construite dans les médias : d'abord l'expression des émotions, puis de l'hommage et enfin du deuil. Pierre Nora définit les manifestations postattentats de janvier 2015 comme un nouvel « événement monstre », dont l'apogée résiderait dans les rassemblements du 11 janvier. Il les analyse ainsi : 
Un surgissement de l'inattendu auquel les médias donnent une répercussion explosive. [...] Des événements à caractère total dans lesquels la communauté se contemple et se réfléchit dans un flamboiement éphémère où se joue quelque chose d'essentiel à son fonctionnement. (Nora, 2015, p. 4)

Même si le temps médiatique réservé aux hommages semble plus court après les attentats de Nice, en dépit du deuil national, les mêmes formes de recueillement qu'en novembre 2015 se succèdent néanmoins. C'est le temps de "L'hommage aux victimes» $(A e F)$, où « des milliers de passants recouvrent les traces de sang de fleurs et d'hommages », mais aussi le temps des avertissements face à la désunion politique et civile : «Soyons à la hauteur» (LMon).

Après l'expression très médiatisée des émotions, viennent cependant la résistance et la résilience. En janvier 2015, la résistance se traduit par une solidarité collective, les rassemblements dans la rue dès le 7 janvier et les soutiens sur internet et les réseaux sociaux. En novembre 2015, les manifestations spontanées, concrétisant «le besoin d'être ensemble, de se serrer» $(A e F)$, sont rattrapées par la logique politique et l'état d'urgence. La résistance se joue dans les tribunes que certains médias offrent aux membres des différentes communautés religieuses (musulmane, catholique et juive) : «Résistons ensemble» $(A e F)$. À Nice, la résistance s'exprime dans la volonté de la société civile de demeurer « unie » et de « rester vigilant[e]». Il s'agit de savoir « comment combattre la barbarie? » (LSoir), « comment tenir?» (LPO), d'envisager « une riposte sans pitié » (LFig). La résilience consiste à poursuivre sa vie d'avant, malgré les menaces et les attaques. Elle se dit surtout après les attentats du 13 novembre et ceux de Nice, du fait de la permanence de la menace terroriste. Après le 13 novembre, les médias montrent une société civile qui refuse de «céder au chantage» $(A e F)$, et « Paris qui se réveille et rouvre ses lieux culturels» (LMon). Les journaux construisent ainsi une forme d'injonction à vivre normalement : «Il va falloir apprendre à vivre avec la mort» (LMon). Plusieurs médias disent aussi la résilience après l'attentat de Nice : Aujourd'hui en France y consacre sa une du 17 juillet («La vie plus forte que la mort») et plusieurs articles («la vie reprend coûte que coûte », « la vie malgré tout»). C'est aussi accepter l'idée d'un avant et d'un après les attentats : «on ne peut pas vivre dans un état de stress permanent» $(A e F)$. Un éditorial du Soir le proclame : c'est «le début d'une vie différente, d'une société plus lourde, plus fermée, plus dure, moins insouciante, et peut-être, c'est une grosse crainte, plus haineuse».

\section{Les auteurs des violences : de "l'étrangéité » à l'ennemi intérieur}

Face à ces sociétés meurtries, et dans une tension narrative classique, les médias organisent leurs récits en revenant sur le parcours des auteurs des vio- 
lences; parcours auxquels s'articulent les tentatives de définition des causalités possibles des actes terroristes. Entre 1995 et 2016, les figures narratives des auteurs évoluent très nettement.

\section{Saint-Michel et Port-Royal : «l'étrangéité » radicale des auteurs}

Dans la décennie 1980, les attentats avaient été imputés à des figures du terrorisme international (Carlos, par exemple). Les attaques de 1995 et 1996 s'inscrivent dans une chronologie terroriste qui fait de leurs auteurs des figures de l'altérité, étrangères à la Nation. Mais pour ces deux attentats, pas de revendication claire ; la qualification des auteurs se fonde sur des fantômes. En juillet 1995 et en décembre 1996, les journaux évoquent cependant tous la «piste islamiste» (et la « piste serbe » pour 1995), qui est «privilégiée» en 1996. Lors de l'attentat de Saint-Michel, les quotidiens mentionnent tous «une» revendication «du GIA - Groupe islamique armé » et des «islamistes algériens». Libération et Le Monde évoquent la «piste intégriste"; Libération réaffirme «l'hypothèse » selon laquelle «l'attentat du RER a [...] des commanditaires, sinon forcément des exécutants, étrangers ». Un article (Bloc-notes) du Point, signé Bernard-Henri Lévy, se focalise sur la figure du "poseur de bombes", "salopard», se "réjouissant» de ses «visées barbares». Il faut noter la mention par Sud Ouest d'une «violence présente [...] dans nos banlieues», tandis que plusieurs quotidiens pointent l'inquiétude des Parisiens «d'origine algérienne » qui craignent «l'amalgame» (LMon, Lib). France-Soir précise que "[l]es islamistes avaient prévenu» et que "[d]eux témoignages accusent les "barbus" ». Le Figaro, prolixe sur les «barbus», interroge cependant le recteur de la Grande Mosquée de Paris, Dalil Boubakeur, qui évoque la menace pesant sur sa «communauté».

En décembre 1996, les journaux rappellent que le «mode opératoire » est le même qu'en 1995 et qualifient l'attentat d' «islamiste». Le terme est très utilisé en 1996, associé aux substantifs «menace», «terrorisme», « réseau», «prosélytisme», «militant», «tueur», «commando» ou «mouvance». La figure des auteurs se dessine, les médias rappellent le démantèlement du réseau responsable de l'attentat de 1995 et supposent des auteurs «fanatisés». Le Figaro identifie la «méthode islamiste» et les «banlieues sous surveillance», affirmant que le «drame pose à nouveau la question du prosélytisme islamiste dans les banlieues et les prisons» - motif discursif qui revient dans les éditions suivantes, accompagné de photographies de suspects potentiels, barbus et inquiétants. France-Soir décrit les "assassins", «terroristes algériens », et indique, sous le titre «Des beurs à la barre des accusés», qu'une «vingtaine d'entre eux, recrutés en banlieue par les islamistes [est] jugée lundi à Paris ». En 1996, le quotidien populaire établit donc plus vite la figure du mal et dénonce en titre cette "Europe si douce aux "fous d'Allah"». 
Les attentats de la décennie 1990 font donc émerger une figure des auteurs du terrorisme qui se précise, en 1996, comme un ennemi agissant à l'intérieur, mais dont l'appartenance à un pays étranger demeure signifiée. Les banlieues sont évoquées, mais les soupçons se focalisent sur un «commando d'Afghans», de jeunes gens formés à l'étranger. Le modèle sociopolitique français et ses lieux d'étrangéité (les banlieues, les prisons) sont interrogés par certains journaux, proches du gouvernement ou populaires. Dans leur ensemble, pourtant, les discours tiennent à distance la figure des auteurs et la construisent comme une figure extérieure, sur les plans territorial ou symbolique, et agressant une nation dont le modèle social n'est pas remis en cause.

\section{Charlie Hebdo, 13 novembre et Nice : des ennemis de l'intérieur au terroriste "hors-cadre»}

Les attentats survenus en 2015 et 2016 remettent en cause les représentations d'un terroriste "exogène », présentes en 1995 et 1996. Comment, dès lors, les médias peuvent-ils expliquer les actes barbares d'individus qui ont grandi dans la République française ou au royaume de Belgique? Où est l'altérité des frères Kouachi? Celle des frères Abdeslam? De Samy Amimour?

La qualification des terroristes évolue par ailleurs avec les modes opératoires : en janvier 2015, il s'agit de «tueurs» et de "criminels», en novembre de «terroristes» et de "kamikazes». Il est par ailleurs notable que, face à la nationalité française de certains des auteurs, la plupart des journaux proposent d'autres éléments d'altérité : ce sont «deux frères connus de la justice », des délinquants passés «du braquage au terrorisme» $(A e F)$, dont la situation familiale peut aussi être évoquée, l'un des auteurs est «orphelin dès l'enfance de ses deux parents immigrés d'Algérie [...] élevé dans un foyer» (Lib). En novembre, les terroristes sont de nationalité belge, française et syrienne. Leur "petit parcours de délinquant» est mis en avant, mais les témoignages des rescapés disent leur «normalité » : «des mecs de 25-30 ans, petite barbichette, pas du tout le look salafiste». L'ambivalence de la figure des terroristes est forte : leur visage ordinaire est contredit par l'image construite par les médias, celle de «combattants aguerris» (LMon), qui n'agissent plus en «loups solitaires » mais en «meute organisée» (Lib).

L'attentat de Nice remet partiellement en cause cette figuration médiatique. Certes, Mohamed Lahouaiej-Bouhlel est tunisien, donc étranger, mais sa radicalisation islamiste est marginale et récente. Les quotidiens éprouvent des difficultés à le décrire par la figure «archétypale» du terroriste islamiste. Le tueur est présenté comme un monstre (il bat sa femme, est condamné pour violences), mais surtout comme un fou - ce qui pourrait le rendre irresponsable de ses actes. Libération comme Le Figaro hésitent à le qualifier de «terroriste islamiste» et présentent une figure inhabituelle : «troubles psycholo- 
giques », «fou», «dragueur», «buveur». L'auteur de la tuerie a un profil de "tueur de masse psychotique», de "suicidaire narcissique» (Lib) et Le Figaro parle de «terroriste hybride». Le Monde revient sur ce profil avec la même interrogation : "Se pourrait-il que l'auteur de l'attentat le plus meurtrier [...] ne soit pas un terroriste? » Les journaux étrangers se posent les mêmes questions: «Terroriste djihadiste ou tueur de masse bipolaire?» (LSoir), et dressent le portrait d'un homme violent mais pas d'un terroriste islamiste - «He never went to the local mosque, often grunted in response to greetings of "bonjour" and sometimes beat his wife - until she threw him out» (NYT). Le tueur de Nice sort donc du cadre des représentations médiatiques de la figure du terroriste interne, présente depuis 2012 (avec Mohamed Merah) et, face à ce profil atypique, le récit ne peut organiser la même opposition de figures narratives. Dans les récits médiatiques, le chagrin social reste donc, partiellement, face à un vide explicatif.

Que nous permet de conclure, brièvement, ce regard rétrospectif porté sur cette longue série d'attentats et de discours médiatiques?

Tout d'abord, que la prise en charge politique des attentats a évolué vers des registres nettement plus guerriers, parfois décrits comme inadéquats par les médias, mais largement étayés par ces mêmes discours médiatiques. Face à la violence terroriste, la fermeté du pouvoir apparaît toujours comme la garantie - toute hobbesienne - de la survie de l'ensemble sociopolitique.

La société civile est, quant à elle, racontée de façon très différente dans les attentats de 2015 et 2016. Ses émotions constituent un motif discursif majeur, structurant, qui permet aux médias de dire la communion face à la douleur et l'unité d'une société atteinte dans sa chair. Les discours de 1995 et 1996 n'abordent pas cette dimension, sauf dans un journal populaire comme FranceSoir, qui propose un discours empreint de sensations. Les représentations contemporaines des attentats font ainsi émerger beaucoup plus fortement qu'il y a 20 ans, dans les espaces éditoriaux, les sentiments, les chagrins et les colères. Et si cela s'explique par l'ampleur des attentats de 2015 et 2016, il faut remarquer cette répercussion symbolique plus importante dans les discours portant sur la société civile. Dans les discours médiatiques les plus contemporains, la guerre du pouvoir est ainsi liée à la douleur partagée de la société.

Enfin, la figure narrative des auteurs des violences a évolué depuis 20 ans. La délégitimation de leurs actes ne peut plus simplement passer par le filtre de l'altérité et de l'islamisation, puisque désormais la menace vient de l'intérieur et implique, comme le précise le New York Times, la nécessité pour l'État français de repenser ce qui est aussi devenu un problème social et politique. Et, face à ce défi, les médias divergent fortement, comme cela apparaît dans les articles publiés une fois clos le temps chaud de la violence. 


\section{Références}

BACOT Paul, Douzou Laurent, HonorÉ Jean-Paul, 2008, «Chrononymes. La politisation du temps", Mots. Les langages du politique, n87, p. 5-12.

BousSAgUET Laurie, FAUCHER Florence, 2016, «Mobiliser des symboles pour répondre au terrorisme : l'exécutif français face aux attentats de 2015 à Paris ", LIEPP Policy Brief, nº 28.

DAYAN Daniel éd., 2006, La terreur spectacle : terrorisme ettélévision, Bruxelles, De Boeck. DERRIDA Jacques, HABERMAS Jürgen, 2004, Le «concept» du 11 septembre : dialogues à New York (octobre-décembre 2001) avec Giovanna Borradori, Paris, Galilée.

FOUCAULT Michel, 1975, Surveiller et punir, Paris, Gallimard.

GARCIN-MARRou Isabelle, 2018, "Les enjeux sociopolitiques des discours des médias sur le terrorisme», dans L'État et le terrorisme, J. Blanc et P. Bourdon éd., Paris, Publications de la Sorbonne, p. 257-276.

- 2001, Terrorisme, médias et démocratie, Lyon, Presses universitaires de Lyon.

GIRARD René, 1994, La violence et le sacré, Paris, Hachette.

GUIGo Pierre-Emmanuel, 2016, «L'étoffe présidentielle à l'épreuve : la communication de F. Hollande pendant les attentats ", dans Le défi Charlie : les médias à l'épreuve des attentats, P. Lefébure et C. Sécail éd., Paris, Lemieux éditeur, p. 79-114.

HARE Isabelle, 2016, "Médias et terrorisme : entre changement et continuité? », Les Cahiers de la SFSIC, nº12, p. 41-52.

- 2007, Représentations médiatiques et stratégies discursives dans les conflits nordirlandais et israélo-palestinien sur Internet et dans la presse écrite, thèse de doctorat, Lyon, Université de Lyon.

Hовв ES Thomas, 1971 [1651], Léviathan, Paris, Vrin.

KOREn Roselyne, 1996, Les enjeux éthiques de l'écriture de presse et la mise en mots du terrorisme, Paris, L'Harmattan.

LEFÉBURE Pierre, SÉCAIL Claire éd., 2016, Le défi Charlie : les médias à l'épreuve des attentats, Paris, Lemieux éditeur.

LITS Marc éd., 2004, Du 11 septembre à la riposte : les débuts d'une nouvelle guerre médiatique, Bruxelles, De Boeck.

NiemeYer Katharina, 2011, De la chute du mur de Berlin au 11 septembre 2001 : le journal télévisé, les mémoires collectives et l'écriture de l'histoire, Lausanne, Antipodes.

NorA Pierre, 2015, «Retour sur un événement monstre », Le Débat, nº 185, p. 4-10.

OrY Pascal, 2016, Ce que dit Charlie : treize leçons d'histoire, Paris, Gallimard.

SCHLESINGER Philip, 1991, Media, State and Nation, Londres, Sage.

TÉTu Jean-François, 2004, "L'émotion dans les médias : dispositifs, formes et figures », Mots. Les langages du politique, $\mathrm{n}^{\circ} 75, \mathrm{p} .9-19$.

TRUC Gérôme, 2016, Sidérations : une sociologie des attentats, Paris, PUF.

VENIARD Marie, 2018, «La presse devant les attentats terroristes: usages journalistiques du mot guerre (Paris, 2015) », Mots. Les langages du politique, n ${ }^{0} 116$, p. 91-109.

Wieviorka Michel, Wolton Dominique, 1987, Terrorisme à la une : média, terrorisme et démocratie, Paris, Gallimard.

Wrona Adeline, 2005, «Vies minuscules, vies exemplaires : récit d'individu et actualité. Le cas des portraits of griefs parus dans le New York Times après le 11 septembre $2001 »$, Réseaux, nº132, p. 93-110. 


\section{Résumé / Abstract / Compendio}

\section{Discours médiatiques post-attentats : une perspective historique (1995-2016)}

La réitération des actes terroristes sur le sol européen depuis 2015 a conduit à interroger la permanence des relations entre État, société civile et médias, à questionner les enjeux sociopolitiques des discours des médias. Ce sont les discours médiatiques relatifs à l'illégitimité de la violence terroriste et à la légitimité des actions de réparation que la contribution entend étudier en proposant une perspective diachronique depuis l'attentat de juillet 1995 à Saint-Michel à Paris jusqu'à l'attentat de Nice en juillet 2016.

Mots-clés : terrorisme, médias, État, société civile, histoire

\section{Media narratives after terrorist attacks: a historical perspective (1995-2016)}

The succession of terrorist acts on European ground since 2015 has led to questioning the permanence of relations between State, civil society and the media, and to questioning the social-political stakes of media narratives. The media narratives relative to the illegitimacy of terrorist violence and the legitimacy of reparation actions are studied in this contribution, which proposes a diachronic perspective from the attack of July 1995 in Saint-Michel, Paris, to the attack of Nice in July 2016.

Keywords: terrorism, media, State, civil society, history

\section{Discursos mediáticos post-atentados: una perspectiva (1995-2016) histórica}

La reiteración de los actos terroristas sobre el suelo europeo desde el 2015 condujo a interrogar la permanencia de las relaciones entre Estado, sociedad civil y medios de comunicación, a indagar las puestas sociopolíticas de los discursos en los medios de comunicación. Son los discursos mediáticos relativos a la ilegitimidad de la violencia terrorista y a la legitimidad de las acciones de reparación lo que esta contribución piensa estudiar, proponiéndose una perspectiva diacrónica desde el atentado de julio de 1995 en Saint-Michel, París hasta el atentado de Niza en julio de 2016.

Palabras claves: terrorismo, medios de comunicación, Estado, sociedad civil, historia 